\title{
A two-stage genome-wide association study of radiation-induced acute toxicity in head and neck cancer
}

\author{
Elnaz Naderi ${ }^{1,2^{*}}$, Anne Petra Gerarda Crijns ${ }^{1}$, Roel Johannes Henricus Marinus Steenbakkers' ${ }^{1}$, \\ Johanna Geertruida Maria van den Hoek', Hendrika Marike Boezen², Behrooz Ziad Alizadeh ${ }^{2 \dagger}$ and \\ Johannes Albertus Langendijk ${ }^{1 *+}$
}

\begin{abstract}
Background: Most head and neck cancer (HNC) patients receive radiotherapy (RT) and develop toxicities. This genome-wide association study (GWAS) was designed to identify single nucleotide polymorphisms (SNPS) associated with common acute radiation-induced toxicities (RITS) in an HNC cohort.

Methods: A two-stage GWAS was performed in $1279 \mathrm{HNC}$ patients treated with RT and prospectively scored for mucositis, xerostomia, sticky saliva, and dysphagia. The area under the curve (AUC) was used to estimate the average load of toxicity during RT. At the discovery study, multivariate linear regression was used in 957 patients, and the topranking SNPs were tested in 322 independent replication cohort. Next, the discovery and the replication studies were meta-analyzed.

Results: A region on 5 q21.3 containing 16 SNPs showed genome-wide (GW) significance association at P-value $<5.0 \times 10^{-8}$ with patient-rated acute xerostomia in the discovery study. The top signal was rs35542 with an adjusted effect size of $0.17^{*} \mathrm{~A}\left(95 \% \mathrm{Cl} 0.12\right.$ to 0.23 ; P-value $<=3.78 \times 10^{-9}$ ). The genome wide significant SNPs were located within three genes (EFNA5, FBXL17, and FER). In-silico functional analysis showed these genes may be involved in DNA damage response and co-expressed in minor salivary glands. We found 428 suggestive SNPs $\left(P\right.$-value $\left.<1.0 \times 10^{-5}\right)$ for other toxicities, taken to the replication study. Eleven of them showed a nominal association (P-value <0.05).

Conclusions: This GWAS suggested novel SNPs for patient-rated acute xerostomia in HNC patients. If validated, these SNPs and their related functional pathways could lead to a predictive assay to identify sensitive patients to radiation, which may eventually allow a more individualized RT treatment.
\end{abstract}

Keywords: Radiogenomics, GWAS, Radiation-induced toxicity, Head and neck cancer

*Correspondence: e.naderi@umcg.nl; j.a.langendijk@umcg.nl

${ }^{\dagger}$ Behrooz Ziad Alizadeh and Johannes Albertus Langendijk have contributed equally

${ }^{1}$ Department of Radiation Oncology, University Medical Center Groningen, Hanzeplein 1, HPC; DA 30, P.O. Box 30 001, 9700 RB Groningen, The Netherlands

Full list of author information is available at the end of the article

\section{Background}

Head and neck cancer (HNC) is a life-threatening disease affecting approximately 650,000 new patients and causing 330,000 deaths annually worldwide [1]. More than $90 \%$ of HNC are squamous cell carcinomas affecting the mucosal membranes, salivary glands, swallowing muscles, craniofacial bones, and soft tissues. original author(s) and the source, provide a link to the Creative Commons licence, and indicate if changes were made. The images or other third party material in this article are included in the article's Creative Commons licence, unless indicated otherwise in a credit line to the material. If material is not included in the article's Creative Commons licence and your intended use is not permitted by statutory regulation or exceeds the permitted use, you will need to obtain permission directly from the copyright holder. To view a copy of this licence, visit http://creativecommons.org/licenses/by/4.0/. The Creative Commons Public Domain Dedication waiver (http://creativeco mmons.org/publicdomain/zero/1.0/) applies to the data made available in this article, unless otherwise stated in a credit line to the data. 
Curative treatment modalities for HNC include surgery, radiotherapy (RT), chemotherapy, targeted agents, or a combination of these depending on the primary tumour site and locoregional tumour extension [2]. Almost $80 \%$ of HNC patients receive RT at some stage of their treatment [3]. The main aim of RT is to obtain long-term tumour control while minimizing healthy tissue damage. However, RT is associated with collateral damage to the healthy tissues surrounding the tumour, resulting in a broad spectrum of acute and late radiationinduced toxicities (RITs) [4]. Patients receiving RT experience varying levels of toxicity, from minor to severe for a period of a few weeks to even lifetime, hampering patients' quality of life $[5,6]$, such as mucosal inflammation (mucositis), mucosal infections, sticky saliva, dry mouth (xerostomia), chronic pain, decreased voice quality, impaired chewing and swallowing (dysphagia).

The risk of RIT is assessed using normal tissue complication probability (NTCP) models. NTCPs are multivariable prediction models built on radiation dose metrics, clinical factors, and patients' characteristics [7]. The performance of current NTCP-models is suboptimal due to patients' differences in normal tissue radio-sensitivity, determined by underlying genetic susceptibility $[8,9]$. Initial evidence suggests individuals affected by ataxiatelangiectasia and Nijmegen Breakage Syndrome, two genetic disorders, are hypersensitive to radiation and unable to undergo RT [10]. Nevertheless, the heritability of radio-sensitivity has not been thoroughly investigated. Some studies based on flow cytometric assays estimated a heritability of 58 to $78 \%$ for cell response to irradiation, which was, in turn, a proxy for radio sensitivity [11-13]. Likewise, two previous studies showed associations between single nucleotide polymorphisms (SNPs) in XRCC1, RAD51, and NBN genes with the development of grade $\geq 2$ radiation-induced mucositis, dysphagia, and skin erythema in HNC patients [14, 15]. Recently, several genome-wide association studies (GWAS) have identified SNPs associated with RITs in breast and prostate cancer patients $[8,16]$. For HNC, a recent GWAS observed significant associations between three SNPs on chromosome 5 with acute mucositis (Line M H Schack et al. under review). Another GWAS found 50 suggestive loci associated with mucositis in Chinese HNC patients [17]. The underlying genetic causes of RITs remain undiscovered yet. We performed a two-stage GWAS in a Dutch HNC cohort to identify SNPs and subsequent potential genetic pathways associated with acute RITs.

\section{Methods}

An extended description of the methods and results can be found in the Additional file 1: Appendix.

\section{Study design}

We performed a two-stage GWAS. At discovery study, we aimed to identify candidate SNPs associated with acute RIT in HNC patients. At the replication study, we sought to confirm the identified discovery SNPs in independent HNC cohort. To gain more statistical power for discovery, we combined discovery and replication cohorts and performed a GWAS for acute RIT (i.e., combined analysis).

\section{Participants}

We included 1429 HNC patients (1102 for the discovery; 327 for the replication study). Patients were treated with primary or postoperative RT either with or without chemotherapy from 2007 to 2020 as part of the UMCG-HNC prospective data registration program (NCT02435576, clinicaltrials.gov). The prospective data registration program has been reviewed by the medical ethical committee and is considered standard of care. Additional written informed consent was obtained for the genetic study (NCT02489084). Data on baseline patient-, tumour-, and treatment- characteristics were collected before starting RT. Additionally, physician and patient-rated acute RITs were prospectively assessed weekly during RT and after completion of RT, up to 7 weeks (called acute RITs)[18].

\section{Assessment of RITs}

Physician-rated acute toxicities, including mucositis, dysphagia, xerostomia, and sticky saliva, were registered according to the Common Toxicity Criteria of Adverse Events (version 4.0) [19]. Patient-rated HNC symptoms were assessed using the EORTC QLQH\&N35 questionnaire in addition to the EORTC QLQC30 [20] (Additional file 1: Table S1).

\section{Multiple imputations of missing value for toxicity}

We observed varying percentages of missingness across RITs (Additional file 1: Table S2). We applied multiple imputation (MI) as implemented in MICE package [21] (Additional file 1: Methods).

\section{Genotyping, quality control, and imputation}

Samples were genotyped using the Illumina human core and GSA arrays. We applied standard participant and SNP level quality control (QC). We checked the ethnicity of participant using multidimensional scaling clustering using EIGENSTRAT. Missing genotypes 
were imputed based on the HRC reference panel (Additional file 1: Supplementary Methods).

\section{Clinical factors}

Based on clinical relevance confirmed by a panel of experts of the Radiogenomics Consortium[22], age, gender, type of RT, concomitant chemotherapy, tumoursite, and baseline toxicity were included as co-variables (Table 1). We added the volume surrogate as defined by Volume $1=\mathrm{T} 1 \mathrm{a}-1 \mathrm{bN} 0 \mathrm{M} 0$ glottic laryngeal carcinomas, Volume $2=$ all other TxN0 sites, and Volume $3=\mathrm{TxN} 1-3$ carcinomas (Line M H Schack et al. under review).

\section{Data analysis}

\section{Outcome modelling}

We assessed RITs using two scoring systems. First, since acute RIT generally increases during treatment, we estimated the area under the curve (AUC) to generate an overall measure of acute toxicity during RT treatment up to seven weeks per each of the RITs (Additional file 1: Supplementary methods). A mean of AUCs represents an average of toxicity per week during the RT plan. Second,

Table 1 Baseline characteristics of included HNC patients

\begin{tabular}{|c|c|c|c|}
\hline Overall N & $\begin{array}{l}\text { Discovery } \\
\mathrm{N}=957\end{array}$ & $\begin{array}{l}\text { Replication } \\
\mathrm{N}=322\end{array}$ & $\begin{array}{l}\text { Total } \\
\mathrm{N}=1279\end{array}$ \\
\hline Period of treatment & 2007-2018 & $2018-2020$ & $2007-2020$ \\
\hline Gender; Female (\%) & $313(32.71)$ & $98(30.40)$ & $411(32.10)$ \\
\hline Mean age (SD) & $63.50(11.75)$ & $65.70(11.63)$ & $63.90(11.75)$ \\
\hline \multicolumn{4}{|l|}{ Age group (\%) } \\
\hline$\geq 55$ & 209 (21.84) & $58(18.00)$ & $267(20.90)$ \\
\hline $56-69$ & $455(47.54)$ & $131(40.70)^{*}$ & $586(45.80)$ \\
\hline $70 \geq$ & $293(30.62)$ & $133(41.30)^{* * *}$ & $426(33.30)$ \\
\hline \multicolumn{4}{|l|}{ Tumor site (\%) } \\
\hline Oral cavity & $180(18.95)$ & $47(14.70)$ & $227(17.70)$ \\
\hline Oropharynx & $194(20.42)$ & $80(25.10)$ & $274(21.40)$ \\
\hline Larynx & $256(26.95)$ & $82(25.70)$ & $338(26.40)$ \\
\hline Others & $320(33.68)$ & $110(34.50)$ & $430(33.60)$ \\
\hline T-stage; T3, 4 (\%) & $362(37.83)$ & $155(51.70)$ & $517(40.42)$ \\
\hline N-stage; N1-3 (\%) & $456(47.65)$ & $154(51.70)$ & $610(47.49)$ \\
\hline \multicolumn{4}{|l|}{ Chemotherapy (\%) } \\
\hline No chemotherapy & 691 (73.98) & $222(71.20)$ & $913(71.4)$ \\
\hline Concomitant chemo & $227(24.30)$ & $79(25.30)$ & $306(23.90)$ \\
\hline Radio + cetuximab & $16(1.72)$ & $11(3.50)$ & $27(2.10)$ \\
\hline \multicolumn{4}{|l|}{ Radiotherapy } \\
\hline Postoperative (\%) & 409 (42.74) & 115(36.30) & $524(40.97)$ \\
\hline \multicolumn{4}{|c|}{ Volume Surrogate } \\
\hline Glottic/laryngeal T1NOMO & $123(13.57)$ & $25(8.60)^{*}$ & $148(11.60)$ \\
\hline All other TxNO sites & $329(36.32)$ & $111(38.30)$ & $440(34.40)$ \\
\hline TxN1-3 carcinomas & $454(50.11)$ & 154(53.10) & $608(47.50)$ \\
\hline
\end{tabular}

*Significance difference between discovery and replication cohorts: ${ }^{*} \mathrm{P}<0.05$ ${ }^{* *} \mathrm{P}<0.01 ;{ }^{* * * P}<0.001$ to achieve a composite score representing the overall acute RIT, we used the standardized total average toxicity (STAT) [23]. STAT ${ }_{\text {physician }}$ and STAT patient included the spectrum of the physician and patient-rated acute RITs, respectively.

\section{Association analyses}

Multivariate linear regression was used to estimate the association of the additive effect of SNPs effect allele with AUCs and STAT scores. Multivariable models were adjusted for the aforementioned covariates and the top 10 PCA eigenvectors. SNPs were included as the number or dosage of effect alleles in (imputed) genotypes resulting in a regression coefficient for one copy increase in effect allele. A genome-wide P-value $<5.0 \times 10^{-8}$ was considered statistically genome-wide significant (GWS), and a $\mathrm{P}$-value $<1.0 \times 10^{-5}$ was considered a suggestive association.

\section{Replication study and meta-analysis}

The SNPs with a suggestive association identified in the discovery study were included in the replication study. We used the same co-variables. Each suggestive SNP was tested for the association with its corresponding RIT. A Bonferroni corrected P-value at $0.05 /$ number of independent loci was considered as statistically significant replication. Per each SNP, the summary statistics of discovery and replication studies were meta-analysed using an inverse variance-weighted fixed-effect model implemented in METAL (version 2011-03-25) [24]. Any SNP with a meta-P-value of $1.0 \times 10^{-5}><5.0 \times 10^{-8}$ was considered as a suggestive SNP and with a meta-Pvalue $<5.0 \times 10^{-8}$ as replicated GWS SNP.

\section{Combined study}

We combined discovery and replication cohorts $(1,429$ HNC patients) performed a GWAS for acute RIT to gain more statistical power for discovery.

\section{Power analysis}

Quanto software [25] was used to estimate the study's statistical power. The study had $80 \%$ power to detect SNPs with effect allele frequencies $>0.45$ and with an effect size of 2.0 or higher at $\mathrm{P}$-value $<5.0 \times 10^{-8}$.

\section{In-silico functional analysis}

To understand the functional effects of the identified genome-wide associated SNPs in the discovery study (GWS $_{\text {discovery }}$ SNPs), we performed an in-silico functional analysis. First, we used Ensembl [26] (release 98) to extract characteristics of the GWS discovery $_{\text {SNPs. Next, }}$ we used SCREEN [27] to explore if the GWS discovery $_{\text {. }}$ SNPs compose cis-Regulatory Elements (ccREs), which 
regulate the expression of nearby genes. Then, we investigated whether ccREs were related to functional elements. We selected genes associated with ccREs and $\mathrm{GWS}_{\text {discovery }}$ SNPs using GeneCards (for biological function) and MalaCards (for related diseases) [28]. We performed gene expression analyses using GTEx (v.8) [29] to understand the expression levels of these genes across all tissues. Genes and tissues were clustered using hierarchical clustering, and eventually, tissues with similarity in expression of genes were clustered together.

\section{Results}

\section{Patient characteristics}

Table 1 describes the baseline and clinical characteristics of the included patients. Among 1429 patients with genotyped data, 35 patients were excluded due to QC and 115 patients did not have clinical data. In total, $1279 \mathrm{HNC}$ patients (mean age $63.9(\mathrm{SD} \pm 11.75$ ) years being $67.9 \%$ men) were included in GWAS (Additional file 1: Fig. S2). We obtained 6,334,207 imputed SNPs for 957 HNC patients in the discovery study and 6,563,883 SNPs in 322 HNC patients in the replication study (Additional file 1: Fig. S2 \& Table S4). Figure 1 and Table 2 describe the distribution of acute RITs in the discovery and replication studies. Additional file 1: Table S5 presents the association of predictors with each of the acute RITs.
Table 2 Average score ( \pm SD) of RITs measure by the area under curve $^{\mathrm{a}}$ and STAT acute

Discovery study Replication study

\begin{tabular}{lll}
\hline $\begin{array}{l}\text { AUC of physician-rated xeros- } \\
\text { tomia }\end{array}$ & $1.93( \pm 0.51)$ & $1.84( \pm 0.50)^{2 *}$ \\
$\begin{array}{l}\text { AUC of physician-rated sticky } \\
\text { saliva }\end{array}$ & $1.68( \pm 0.43)$ & $1.73( \pm 0.49)$ \\
$\begin{array}{l}\text { AUC of physician-rated dys- } \\
\text { phagia }\end{array}$ & $1.89( \pm 0.81)$ & $1.73( \pm 0.81)^{2 *}$ \\
AUC of physician-rated mucositis & $1.95( \pm 0.70)$ & $1.90( \pm 0.76)$ \\
STAT physician & $0.02( \pm 0.78)$ & $-0.06( \pm 0.78)$ \\
AUC of patient-rated xerostomia & $2.20( \pm 0.71)$ & $2.21( \pm 0.77)$ \\
AUC of patient-rated sticky saliva & $2.08( \pm 0.72)$ & $2.13( \pm 0.78)$ \\
STAT patient & $0.00( \pm 0.87)$ & $0.02( \pm 0.96)$
\end{tabular}

AUC area under curve; STAT standardized total average toxicity

a Fig. 1 shows details of the distribution of outcomes

*Significance difference between discovery and replication cohorts: ${ }^{*} \mathrm{P}<0.05$ or ${ }^{2 *} \mathrm{P}<0.01$

\section{GWAS}

We found sixteen $\mathrm{GWS}_{\text {discovery }}$ SNPs, tagged one locus, associated with patient-rated acute xerostomia. The $428 \mathrm{GW}$ suggestive SNPs spanned over 117 loci associated with acute RITs (Additional file 1: Fig. S3). Neither the $16 \mathrm{GWS}_{\text {discovery }}$ SNPs, nor the 428 GW suggestive SNPs were significantly associated with their corresponding endpoints in the replication

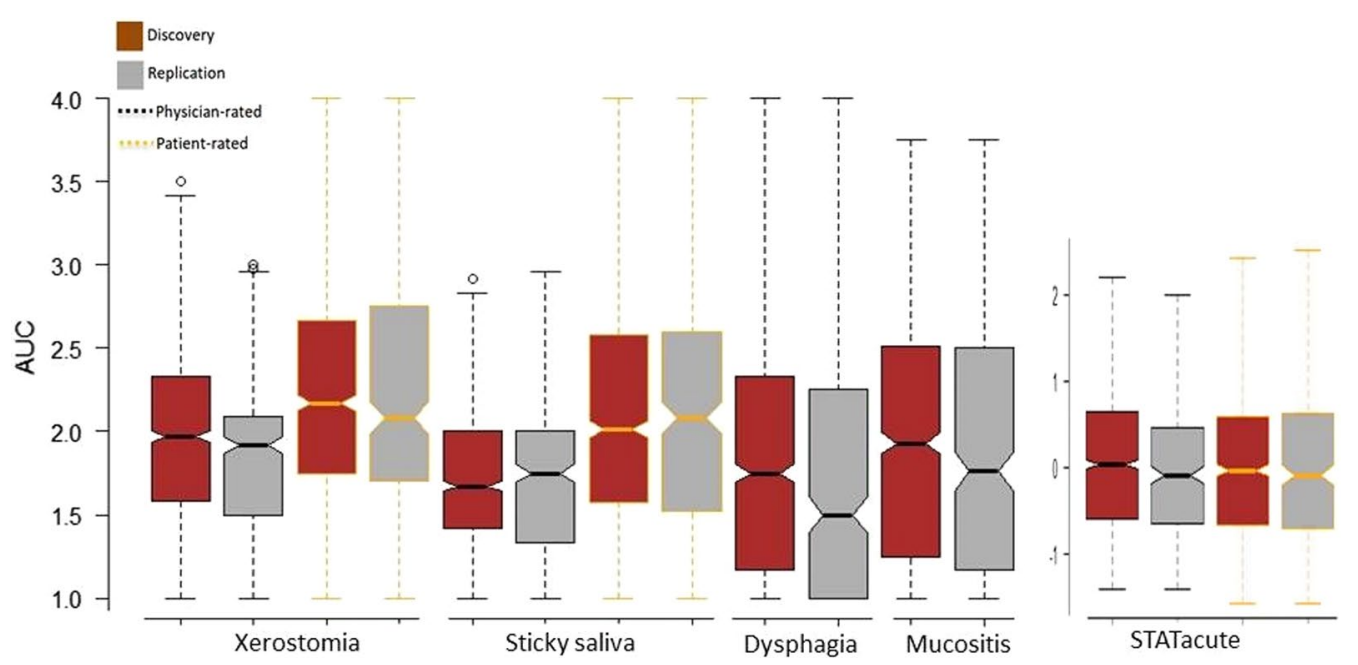

Fig. 1 The dispersion of AUCs and STAT scores of acute radiotoxicity endpoints in HNC patients by discovery and replication cohorts. The X-axis shows the endpoints, and Y-axes show the measured value of the AUC and STAT score of endpoints. The Brown color represents the discovery study, and the grey color represents the replication study. The black border shows physician-rated endpoints, and the orange border shows patient-rated endpoints. The lowest line represents the minimum (Q0 or 0th percentile): the top line represents the maximum (Q4 or 100th percentile) data point excluding any outliers; The middle line represents the median (Q2 or 50th percentile); the box represents the interquartile range (IQR) which is the distance between the first quartile (Q1 or 25th percentile; that is the median of the lower half of the dataset) and the third quartile (Q3 or 75th percentile that is the median of the upper half of the dataset). Table 2 shows the comparison between discovery and replication studies for the significant difference in outcomes' distribution 
study (Additional file 2: Table S6). Eleven out suggestive SNPs showed a nominal association to their corresponding acute RITs (Additional file 2: Tables S7-S14). We found no SNP was significantly associated with any of the eight tested acute RITs in combined analysis. There were $710 \mathrm{GW}$ suggestive SNPs spanned across 137 genomic regions associated with different acute RITs (Additional file 1: Fig. S3).

\section{Patient-rated acute xerostomia}

Among the $16 \mathrm{GWS}_{\text {discovery }}$ SNPs associated with patientrated acute xerostomia in the discovery study (Fig. 2A, B), the top signal was rs35542 with an association effect size (beta) of 0.17 ( $95 \%$ CI 0.12 to 0.23 ; P-value $\leq 3.78 \times 10^{-9}$ ) per increase one copy of A effect allele. These 16 SNPs were in high linkage disequilibrium (LD) (Additional file 1: Fig. S4). Additionally, 43 SNPs from 15 genomic regions were suggestively $\left(\mathrm{P}\right.$-value $<1.0 \times 10^{-5}$ ) associated with patient-rated acute xerostomia. None of the
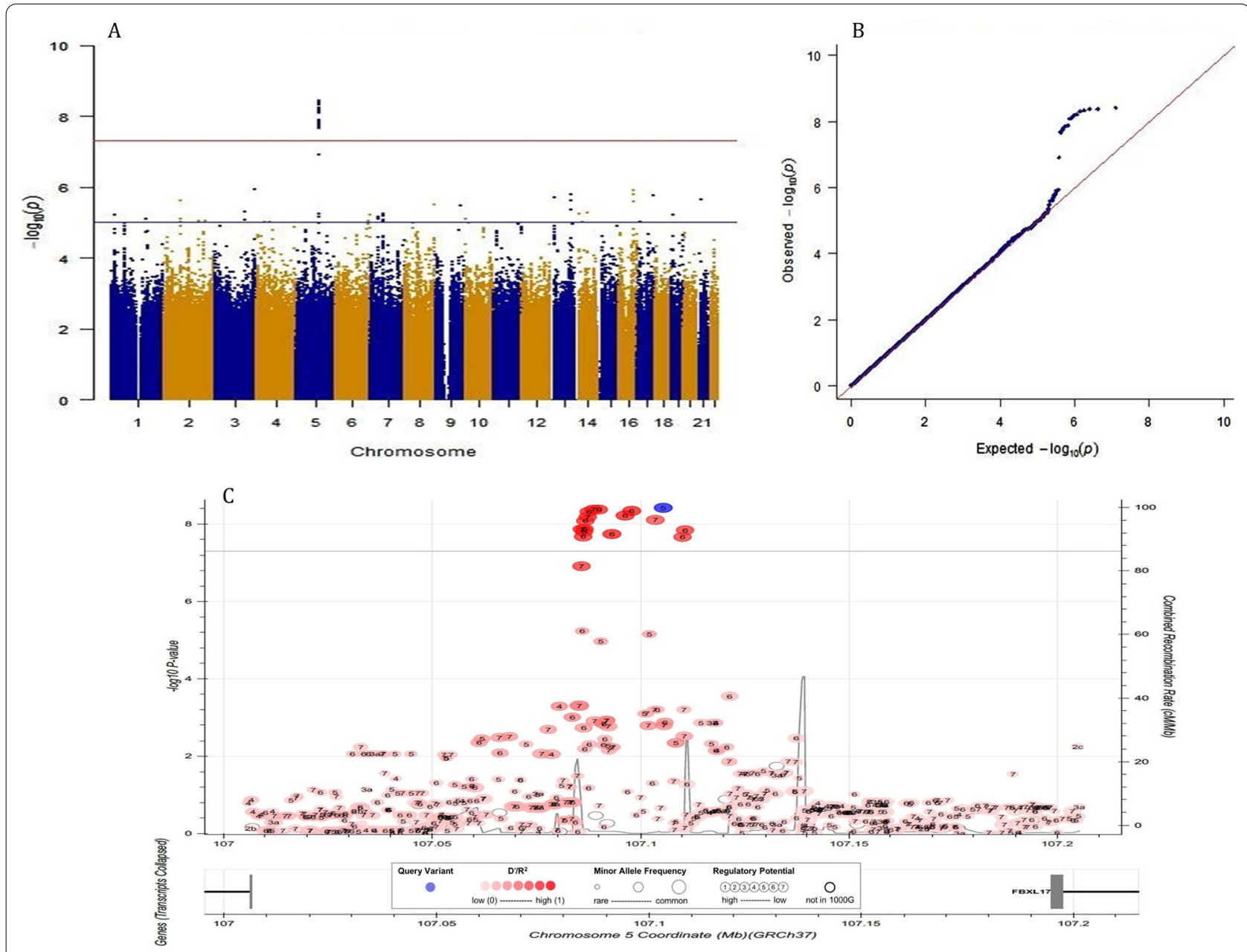

Fig. 2 Genome-wide association findings for AUC for patient-rated toxicity xerostomia in HNC patients. 2A. Manhattan plot: The X-axis shows the location in the genome. The Y-axis shows - $\log 10$ P-values for the association of each of the tested SNPs with the outcome. The red line shows the threshold for genome-wide significance $(P<5 \times 10-8)$, and the blue line shows the suggestive threshold $(P<1 \times 10-5)$. 2B. Quantile-quantile $(Q Q)$ plot comparing the distribution of observed P-values (test statistics) from discovery study to the distribution of expected P-value based on the theoretical probability distribution, inflation of plot to upper part suggest inflation of test statistics due to the possibility of population substructure or type 1 error (small sample size bias). The Y-axis shows observed - $\log 10$ P-values, and the X-axis shows the expected - log 10 P-values. Each SNP is plotted as a dark blue dot, and the red line indicates a null hypothesis of no true association. Deviation from the expected P-value distribution is evident only in the tail area, with a lambda of 1.001, suggesting that population stratification was adequately controlled. 2C. Locuszoom plot of the associated region on chromosome 5. The blue circle (query variant) points to the top SNP (rs35542). Points representing nearby SNPs are color-coded according to linkage disequilibrium $r 2$ value as indicated in the legends. The $X$-axis shows the genomic coordinates chromosome 5. The Y1 axis shows - $\log 10$ P-values for each of the SNPs in the genome. The Y2 axis shows the combined recombination rate which is estimated from the international HapMap project 


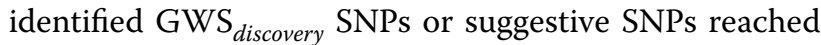
into a statistically significant replication P-value. For the top rs35542 SNP, the meta-analysis reached a metaeffect size of 0.13 ( $95 \%$ CI 0.08 to $0.18 ; \mathrm{P}_{\text {meta }}=5.31 \times 10^{-7}$ (Additional file 2: Table S7).

Additional file 1: Supplementary result and Additional file 2: Tables S7-S14 report the details of variants with a suggestive associations at $\mathrm{P}$-value $<1.0 \times 10^{-5}$ with all endpoints.

\section{In-silico functional analysis}

Annotation analysis showed the GWS discovery $_{\text {SNPs with }}$ patient-rated acute xerostomia were mapped into a noncoding genomic-block (107085963 to 107110731 base pair; GRCh37.p13.chr5) homing to ccREs identified by ENCODE functional dataset. The ccREs were consistently subjected epigenetic activity by histone modification of the H3K27Ac (acylation of lysine 27 of histone 3 ) and H3K4me3 (methylation of lysine 4 of histone 3). The Ephrin-A5 (EFNA5), F-Box and Leucine-Rich Repeat Protein 17 (FBXL17), and FER Tyrosine Kinase (FER) genes are co-localized on the same block with these ccREs, where the $\mathrm{GWS}_{\text {discovery }}$ SNPs reside (Fig. 2C). Using GeneCards and MalaCards, showed EFNA5, $F B X L 17$, and FER have been associated with severe combined immunodeficiency characterized by sensitivity to ionizing radiation disease, DNA damage response after ionizing radiation and activation of the ataxia-telangiectasia mutated protein, respectively. The GTEx tissuespecific expression profiles showed the nearest gene, $E F N A 5$, is highly expressed in minor salivary glands with a median expression level equal to 14.04 TPM (Fig. 3A). Using multi-gene query visualization showed these three genes have the same co-expression pattern in secretory tissues including the minor salivary gland, vagina, and pituitary and also in sun-exposed skin (Fig. 3B).

\section{Discussion}

This GWAS aimed to identify SNPs associated with acute RITs in HNC patients. In the discovery study, we found 16 SNPs associated with patient-rated acute xerostomia at the GWS level. The functional analysis showed plausible biological mechanisms supporting that identified GWS SNPs may play a role in radiation response in healthy tissues generally. However by replication and meta-analysis, our top significant hits shifted up to the suggestive associations. Also, 428 SNPs showed suggestive association with other acute RITs. The majority showed consistent effect directions in discovery and replication studies. By combined analysis, none of the SNPs was GWS, which is likely due to type II error lack of statistical power (i.e. false negative). By combined analysis, we found more suggestive SNPs (710 in 137 loci) associated with acute RITs than two-staged GWAS analysis.

We found a genomic block on 5q21.3 consisting of 16 highly linked (in LD) SNPs associated with patientrated acute xerostomia at the discovery study. The only concurrent GWAS in HNC was by Schack et al. who observed significant associations between three SNPs mapped on chromosome 5(5q31.2) with acute mucositis (Line M H Schack et al. under review). There is no other GWAS analysis of HNC patients in Caucasians. Although a Chinese GWAS found 50 suggestive genomic loci (P-value $<1.0 \times 10-4)$ mapped in 65 genes associated with radiation-induced mucositis in HNC patients [17], but we failed to replicate their findings in our study. However, in breast cancer by using a 2-stage design of 305 SNPs across 59 candidate genes, Seibold et al. reported the association of rs2682585 SNP in the base excision repair gene $X R C C 1$, with late RIT in breast cancer [30]. In a recent meta-analysis of European ancestry cohorts, Kerns et al. identified three SNPs associated with rectal bleeding, decreased urinary stream, and haematuria after RT for prostate cancer [31]. A meta-analysis showed a significant association between the ataxia telangiectasia mutated (ATM) rs1801516*Asn allele with increased risk of radiation-induced tissue toxicity in breast and prostate cancers [16]. Though there were no other GWAs in HNC to be compared, the ATM associated pathway in two cancers seems interestingly linked to the functional analysis of annotation of the $16 \mathrm{GWS}_{\text {discovery }}$ SNPs. These SNPs located across several ccREs bounded to H3K4me3 (methylation of lysine 4 of histone 3). H3K4me3 is implicated in repairing of double-strand DNA breaks (DSBs) caused by ionizing radiation during RT [32, 33]. Furthermore, the neighbouring genes, including EFNA5, $F B X L 17$, and FER, are related to radio-sensitivity. EFNA5 has been associated with severe combined immunodeficiency with sensitivity to ionizing radiation (SCID) disease [34]. EFNA5 was implicated in repairing the DNA damage induced by ionizing radiation, and FBXL17 is involved in U2OS osteosarcoma cellular sensitivity to ionizing radiation [35]. Finally, FER was also involved in activating of $A T M$ protein as a central regulator of DNA damage response after RT. Finally, multi-gene expression visualization showed a high co-expression of EFNA5 and FBXL17 \& FER in the minor salivary gland, suggesting these genes may play a role in the production and secretion of saliva. In summary, carriership of rs $35542 \% \mathrm{~A}$ allele may indicate a dis-regulation of the expression of EFNA5, FBXL17, and FER genes. The dis-regulation, in turn, alters the cell response to enhanced production of reactive oxygen species induced by RT in the minor salivary gland leading eventually to cellular damage and a decrease in the production of saliva, the hallmark of 


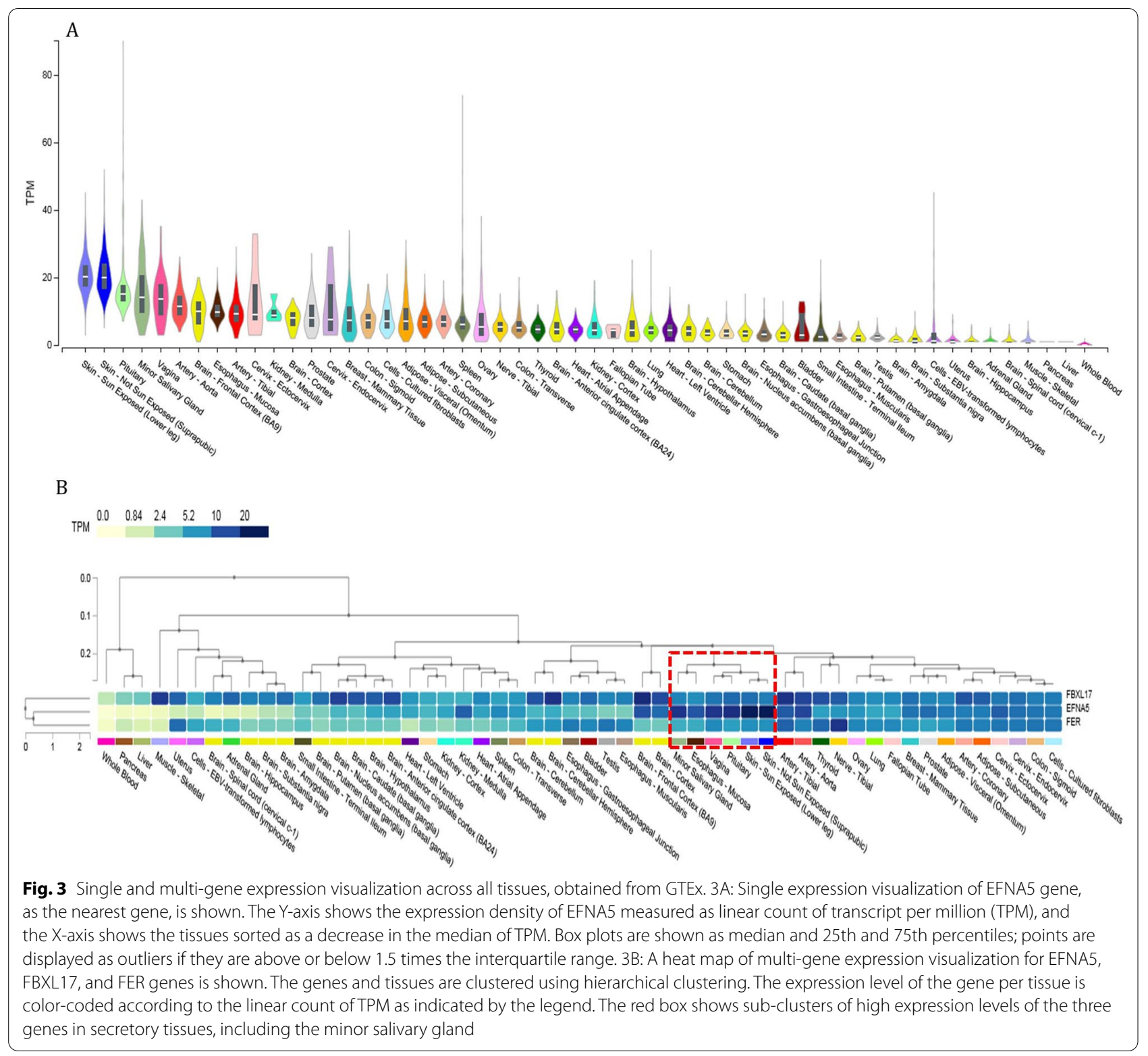

xerostomia. Further research in transcriptome and proteome levels is needed to validate this hypothesis. More details of functional analysis are included in the Additional file 1: Supplementary Results.

Despite convincing functional analysis which supports the genetic finding in discovery study, we were not able to replicate our top hits. In general, non-replication is common in observational studies, mostly attributed to lack of sufficient study power. The nonreplication may be explained by differences in the RT technologies used in the discovery and replication cohorts. The discovery cohort was treated with photon-based techniques, including intensity-modulated radiotherapy (IMRT) or Volumetric Arc Therapy (VMAT). Since 2018, intensity-modulated proton therapy (IMPT) has been clinically introduced in our centre, and patients were either treated with VMAT or IMPT based on predefined selection criteria [36]. Although the prescribed dose for tumour cells remained the same, the exposed dose to the relevant organs-at-risk and the spatial dose distributions per organ were significantly lower with IMPT, this may explain that the results from the discovery study could not be replicated. This is nicely illustrated by the fact that compared to the replication cohort, the discovery cohort showed significantly higher rates of acute RITs. 
Finally, the replication cohort included a limited number of patients with a lower incidence of RITs, which reduced the power of the replication study. Furthermore, the type of RT is likely to be a modifier, as it may change the incidence of RITs; however, this effect is independent of the carriership of genetic variants. Therefore change in the incidence of radiotoxicity due to the type of RT is deemed independent of genetic variation. Therefore, the difference in RT may not significantly modify the association between genetic variants and the studied acute RITs. Future studies with larger sample sizes are needed to determine whether the suggestive SNPs with marginally significant $\mathrm{P}$-values are indeed associated with RITs in HNC patients.

In addition to patient-rated acute xerostomia, we studied seven other outcomes in which we did not find GWS associations, except for several suggestive associations. The lack of finding of GWS to outcomes is common. Previous GWASs have already observed the same phenomena $[16,30]$. For example, Schack et al. studied nine $\mathrm{HNC}$ outcomes and found a single association with acute mucositis (Schack LMH et al. under review) but found no association for the rest of the eight studied outcomes. The differences in the frequency of the endpoints, patient selection, study setting, and treatment modalities reduce study power of GWAS for radiotoxicity.

This study has several strengths. HNC patients were selected out of a well-characterize prospective cohort, which is treated according to predefined guidelines over several years; by which radiation dosage was carefully assessed, the patient's clinical response and side effect were systematically collected. The extent of acute RIT was characterized using AUC to estimate the average load of toxicity during the RT treatment period. We accounted for the patient- and treatment-related factors that influence the risk of acute RITs. Multiple imputation approach was used to handle missing values of RITs with high accuracy. This study also has some limitations. First, our modest sample size and subsequently low study power to detect SNPs with small effects. This study had $30 \%$ power to gain the GWS effect of (the rs2682585) SNP with a minor allele frequency (MAF) of 0.4 and an effect size of 1.4 on xerostomia. Second, functional analyses were done using online sources of expression data in a healthy population, as no patient data were available.

The clinical impact of identifying the genetic markers associated with RIT is yet to be defined. One immediate impact is including genetic variants in forming a prediction model that explains patients' sensitivity, preceding RT. The ultimate question is whether the performance of currently used NTCP-models containing both dose and clinical parameters could be improved by addition of SNPs profiles for RIT.

\section{Conclusions}

We identified a locus on 5 q21.3 reaching GWS for association with radiation-induced acute xerostomia in the discovery study; however, we failed to replicate this finding in the replication study, likely due to the complexity of genetic studies in acute RITs, and subsequently the lack of sufficient study power. Nevertheless, in-silico functional analysis showed the region includes several ccREs likely to be involved in co-expression of the EFNA5, FBXL17, and FER genes in minor salivary glands. Therefore, future multicenter larger genetic studies are needed to verify our findings. In vitro/ vivo functional analyses may reveal whether EFNA5FBXL17-FER complex is causally associated with radiation-induced tissue damage in minor salivary glands and hence in the xerostomia pathogenesis.

\section{Abbreviations}

ATM: Ataxia telangiectasia mutated; AUC: Area under the curve; ccRE: Cisregulatory elements; DSB: Double-strand break; GW: Genome-wide; GWAS: Genome-wide association study; GWS: Genome-wide significant; HNC: Head and neck cancer; IMPT: Intensity-modulated proton therapy; IMRT: Intensitymodulated radiotherapy; LD: Linkage disequilibrium; MAF: Minor allele frequency; MI: Multiple imputation; NTCP: Normal tissue complication probability; QC: Quality control; RIT: Radiation-induced toxicity; RT: Radiotherapy; SCID: Sensitivity to ionizing radiation; SNPS: Single nucleotide polymorphisms; STAT: Standardized total average toxicity; VMAT: Volumetric arc therapy.

\section{Supplementary Information}

The online version contains supplementary material available at https://doi. org/10.1186/s12967-021-03145-1.

Additional file 1: Introduction. Material and methods. Results. Table S1: Endpoints. Table S2: Missing values in acute RITs. Table S3.a, b \& c: Pre-imputation quality control genotyped samples. Table S4: Post-imputation quality control. Table S5: Heatmap of association of the predictors with acute RITs. Figure S1: PCA analysis for Ethnicity. Figure S2: Inclusion/exclusion criteria of study patients. Figure S3: Manhattan and QQ plots of GWA results for acute RITs. Figure S4: LD pattern of the 16 GWAs SNPs with patient-rated acute xerostomia. References.

Additional file 2: Table S6: Genome wide significant SNPs asscoiated with patient-rated acute xerostomia. Table S7: Suggestive SNPs associated with patient-rated acute xerostomia. Table S8: Suggestive SNPs associated with physician-rated acute xerostomia. Table S9: Suggestive SNPS associated with physician-rated acute dysphagia. Table S10: Suggestive SNPs associated with patient-rated acute sticky saliva. Table S11: Suggestive SNPs associated with physician-rated acute sticky saliva. Table S12: Suggestive SNPs associated with physician-rated acute mucositis. Table S13: Suggestive SNPs associated with STATphysician. Table S14: Suggestive SNPS associated with STATpatient

\section{Acknowledgements}

We thank all patients who participated in the study and the participating clinic staff for their contribution to data collection.

\section{Authors' contributions}

Study design happened as a collaboration between EN, BZA and LJA. APGC, RJS, JGMH and LJA provided clinical data. EN analysed the data and wrote the manuscript, with contributions from BZ. MHB, BZA and LJA revised the manuscript. All authors read and approved the final manuscript. 


\section{Funding}

Elnaz Naderi is supported by a Ph.D. scholarship from the Graduate School of Medical Sciences, University Medical Center Groningen, The Netherlands. The funder had no role in the design of the study; in the collection, analyses, or interpretation of data; in the writing of the manuscript, or in the decision to publish the results.

\section{Availability of data and materials}

The datasets used and analysed during the current study are available from the corresponding author on reasonable request.

\section{Declarations}

\section{Ethics approval and consent to participate}

All patients were included in the UMCG-HNC prospective data registration program (NCT02435576, clinicaltrials.gov). The prospective data registration program has been reviewed by the medical ethical committee and is considered standard of care. Additional written informed consent was obtained for the genetic study (NCT02489084).

\section{Consent for publication}

Not applicable.

\section{Competing interests}

The authors declare that they have no competing interests.

\section{Author details}

${ }^{1}$ Department of Radiation Oncology, University Medical Center Groningen, Hanzeplein 1, HPC; DA 30, P.O. Box 30 001, 9700 RB Groningen, The Netherlands. ${ }^{2}$ Department of Epidemiology, University Medical Center Groningen, Groningen, The Netherlands.

Received: 13 September 2021 Accepted: 15 November 2021 Published online: 27 November 2021

\section{References}

1. Bray F, Ferlay J, Soerjomataram I, Siegel RL, Torre LA, Jemal A. Global cancer statistics 2018: GLOBOCAN estimates of incidence and mortality worldwide for 36 cancers in 185 countries. CA Cancer J Clin. 2018;68(6):394-424

2. Halmos GB, Bras $L$, Siesling $S$, van der Laan BFAM, Langendijk JA, van Dijk BAC. Age-specific incidence and treatment patterns of head and neck cancer in the Netherlands - a cohort study. Clin Otolaryngol. 2018:43(1):317-24.

3. Brodin NP, Kabarriti R, Garg MK, Guha C, Tomé WA. Systematic review of normal tissue complication models relevant to standard fractionation radiation therapy of the head and neck region published after the QUANTEC reports. Int J Radiat Oncol Biol Phys. 2018;100(2):391-407.

4. Sroussi HY, Epstein JB, Bensadoun RJ, Saunders DP, Lalla RV, Migliorati $C A$, et al. Common oral complications of head and neck cancer radiation therapy: mucositis, infections, saliva change, fibrosis, sensory dysfunctions, dental caries, periodontal disease, and osteoradionecrosis. Cancer Med. 2017;6(12):2918-31.

5. West CM, Barnett GC. Genetics and genomics of radiotherapy toxicity: towards prediction. Genome Med. 2011;3(8):1-15.

6. Pinna R, Campus G, Cumbo E, Mura I, Milia E. Xerostomia induced by radiotherapy: an overview of the physiopathology, clinical evidence, and management of the oral damage. Ther Clin Risk Manag. 2015;11:171-88.

7. Widder J, Van Der Schaaf A, Lambin P, Marijnen CAM, Pignol JP, Rasch CR, et al. The quest for evidence for proton therapy: model-based approach and precision medicine. Int J Radiat Oncol Biol Phys. 2016;95(1):30-6. https://doi.org/10.1016/j.jijrobp.2015.10.004.

8. Herskind C, Talbot CJ, Kerns SL, Veldwijk MR, Rosenstein BS, West CML. Radiogenomics: a systems biology approach to understanding genetic risk factors for radiotherapy toxicity? Cancer Lett. 2016;382(1):95-109. https://doi.org/10.1016/j.canlet.2016.02.035.
9. Andreassen CN, Alsner J, Overgaard J. Does variability in normal tissue reactions after radiotherapy have a genetic basis - where and how to look for it? Radiother Oncol. 2002;64(2):131-40.

10. Gatti RA. The inherited basis of human radiosensitivity. Acta Oncol (Madr). 2001:40(6):702-11.

11. Curwen GB, Cadwell KK, Winther JF, Janet Tawn E, Rees GS, Olsen JH, et al. The heritability of G2 chromosomal radiosensitivity and its association with cancer in Danish cancer survivors and their offspring. Int J Radiat Biol. 2010;86(11):986-95.

12. Roberts SA, Spreadborough AR, Bulman B, Barber JBP, Evans DGR, Scott D. Heritability of cellular radiosensitivity: a marker of low-penetrance predisposition genes in breast cancer? Am J Hum Genet. 2002;65(3):784-94.

13. Finnon P, Robertson N, Dziwura S, Raffy C, Zhang W, Ainsbury L, et al. Evidence for significant heritability of apoptotic and cell cycle responses to ionising radiation. Hum Genet. 2008;123(5):485-93.

14. Pratesi N, Mangoni M, Mancini I, Paiar F, Simi L, Livi L, et al. Association between single nucleotide polymorphisms in the XRCC1 and RAD51 genes and clinical radiosensitivity in head and neck cancer. Radiother Oncol. 2011;99(3):356-61. https://doi.org/10.1016/j.radonc.2011.05.062.

15. Venkatesh GH, Manjunath VB, Mumbrekar KD, Negi H, Fernandes DJ, Sharan $\mathrm{K}$, et al. Polymorphisms in radio-responsive genes and its association with acute toxicity among head and neck cancer patients. PLOS ONE. 2014;9(3):e89079.

16. Andreassen CN, Rosenstein BS, Kerns SL, Ostrer H, De Ruysscher D, Cesaretti JA, et al. Individual patient data meta-analysis shows a significant association between the ATM rs1801516 SNP and toxicity after radiotherapy in 5456 breast and prostate cancer patients. Radiother Oncol. 2016;121(3):431-9.

17. Yang DW, Wang TM, Zhang JB, Li XZ, He YQ, Xiao R, et al. Genome-wide association study identifies genetic susceptibility loci and pathways of radiation-induced acute oral mucositis. J Transl Med. 2020;18(1):1-12.

18. Christianen MEMC, Schilstra C, Beetz I, Muijs CT, Chouvalova O, Burlage FR, et al. Predictive modelling for swallowing dysfunction after primary (chemo)radiation: Results of a prospective observational study. Radiother Oncol. 2012;105(1):107-14. https://doi.org/10.1016/j.radonc.2011.08.009.

19. National Cancer Institute. Common terminology criteria for adverse events (2009). 2014;2009. http://evs.nci.nih.gov/ftp1/CTCAE/CTCAE_4. 03_2010-06-14_QuickReference_5x7.pdf

20. Scott NW, Fayers PM, Aaronson NK, Bottomley A, De Graeff A, Groenvold $M$, et al. Reference Values - This manual presents reference data for the QLQ-C30 based upon data provided by EORTC Quality of Life Memebrs and other users of the QLQ-C30. EORTC Qual Life Study Gr. 2008;(July).

21. van Buuren S, Groothuis-Oudshoorn K. mice: Multivariate imputation by chained equations in R. J Stat Softw. 2011;45(3):1-67.

22. West $C$, Rosenstein BS. Establishment of a radiogenomics consortium. Radiother Oncol. 2010;94(1):117-8.

23. Barnett GC, West CML, Coles CE, Pharoah PDP, Talbot CJ, Elliott RM, et al. Standardized total average toxicity score: a scale- and grade-independent measure of late radiotherapy toxicity to facilitate pooling of data from different studies. Int J Radiat Oncol Biol Phys. 2012;82(3):1065-74.

24. Willer CJ, Li Y, Abecasis GR. METAL: fast and efficient meta-analysis of genomewide association scans. Bioinformatics. 2010;26(17):2190-1.

25. Gauderman J, Morrison J. QUANTO 1.1: A computer program for power and sample size calculations for genetic-epidemiology studies; 2006. http://hydra.usc.edu/gxe

26. Hunt SE, McLaren W, Gil L, Thormann A, Schuilenburg H, Sheppard D, et al. Ensembl variation resources. Database (Oxford). 2018;2018(8):1-12

27. Davis CA, Hitz BC, Sloan CA, Chan ET, Davidson JM, Gabdank I, et al. The encyclopedia of DNA elements (ENCODE): data portal update. Nucleic Acids Res. 2018;46(D1):D794-801. https://doi.org/10.1093/nar/gkx1081.

28. Stelzer G, Rosen N, Plaschkes I, Zimmerman S, Twik M, Fishilevich S, et al. The GeneCards suite: from gene data mining to disease genome sequence analyses. Curr Protoc Bioinf. 2016;16:1.30.1-1.30.33.

29. Manuscript A. The GTEx consortium. The genotype-tissue expression (GTEx) project. Nat Genet. 2013;45(6):580-5.

30. Seibold P, Behrens S, Schmezer P, Helmbold I, Barnett G, Coles C, et al. XRCC1 polymorphism associated with late toxicity after radiation therapy in breast cancer patients. Int J Radiat Oncol Biol Phys. 2015;92(5):1084-92. https://doi.org/10.1016/j.jirobp.2015.04.011.

31. Kerns SL, Fachal L, Dorling L, Barnett GC, Baran A, Peterson DR, et al. Radiogenomics consortium genome-wide association study 
meta-analysis of late toxicity after prostate cancer radiotherapy. JNCl J Natl Cancer Inst. 2019:112:1-12.

32. Wei S, Li C, Yin Z, Wen J, Meng H, Xue L, et al. Histone methylation in DNA repair and clinical practice: New findings during the past 5-years. J Cancer. 2018;9(12):2072-81.

33. Toulany M. Targeting DNA double-strand break repair pathways to improve radiotherapy response. Genes (Basel). 2019;10(1):1-20.

34. Moshous D, Callebaut I, De Chasseval R, Corneo B, Cavazzana-Calvo M, Le Deist F, et al. Artemis, a novel DNA double-strand break repair/V(D)J recombination protein, is mutated in human severe combined immune deficiency. Cell. 2001;105(2):177-86.

35. Hurov KE, Cotta-Ramusino C, Elledge SJ. A genetic screen identifies the Triple T complex required for DNA damage signaling and ATM and ATR stability. Genes Dev. 2010;24(17):1939-50.
36. Tambas M, Steenbakkers RJHM, van der Laan HP, Wolters AM, Kierkels RGJ, Scandurra D, et al. First experience with model-based selection of head and neck cancer patients for proton therapy. Radiother Oncol. 2017;2020(151):206-13. https://doi.org/10.1016/j.radonc.2020.07.056.

\section{Publisher's Note}

Springer Nature remains neutral with regard to jurisdictional claims in published maps and institutional affiliations.
Ready to submit your research? Choose BMC and benefit from:

- fast, convenient online submission

- thorough peer review by experienced researchers in your field

- rapid publication on acceptance

- support for research data, including large and complex data types

- gold Open Access which fosters wider collaboration and increased citations

- maximum visibility for your research: over 100M website views per year

At BMC, research is always in progress.

Learn more biomedcentral.com/submissions 